\title{
Relación entre la motivación al logro y el sentido de propósito con la permanencia de un grupo de madres adolescentes en el sistema educativo costarricense, un aporte desde la orientación ${ }^{1}$
}

\author{
Relation between achievement motivation and sense of purpose with permanence of \\ teenage mothers on the Costa Rican education system, a contribution from counseling ${ }^{2}$ \\ Relação entre motivação para vencer um desafio e sentido de responsabilidade na \\ permanência de um grupo de mães adolescentes no sistema educativo da Costa Rica. Uma \\ contribuição desde a orientação educativa ${ }^{3}$
}

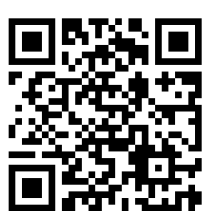

Ester Badilla-Marín ${ }^{4}$

Universidad de Costa Rica

San José, Costa Rica

ester.badilla@ucr.ac.cr

(iD) http://orcid.org/0000-0003-1894-0669

Ana Estrella Meza-Rodríguez ${ }^{5}$

Universidad de Costa Rica

San José, Costa Rica

ana.meza@ucr.ac.cr

(D) http://orcid.org/0000-0001-9509-3438

Recibido 10 de mayo de 2016 • Corregido 20 de junio de 2017 • Aceptado 6 de agosto de 2017

Received 10 de mayo de 2016 • Revised 20 de junio de 2017 • Accepted 6 de agosto de 2017

Recebido 10 de mayo de 2016 • Revisado 20 de junio de 2017 • Aprovado 6 de agosto de 2017

\footnotetext{
${ }^{1}$ El artículo se deriva de la investigación: Rasgos del sentido de vida que favorecen la permanencia de la madre adolescente en el sistema educativo formal (Badilla-Marín et al., 2016).

${ }^{2}$ This article is a derivative of the research: Features of the meaning of life that favor the permanence of teenage mothers in the formal education system.

${ }^{3} \mathrm{O}$ artigo é derivado da pesquisa: Características do sentido de vida que favorecem a permanência da mãe adolescente no sistema de educação formal (Badilla-Marín, Castro-Lopez, 2016).

${ }^{4}$ Bach. en Ciencias de la Educación con énfasis en Orientación de la Universidad de Costa Rica, Lic. en Ciencias de la Educación con énfasis en Orientación de la Universidad de Costa Rica, orientadora en Benemérito Colegio Nocturno José Martí.

${ }^{5}$ Bach. en Ciencias de la Educación con énfasis en Orientación de la Universidad de Costa Rica, Lic. en Ciencias de la Educación con énfasis en Orientación de la Universidad de Costa Rica, estudiante avanzada de la Maestría Académica en Educación con énfasis en Orientación Familiar, docente de la Escuela de Orientación de la Universidad de Costa Rica.
} 
doi: http://dx.doi.org/10.15359/ree.21-3.8

URL: http://www.una.ac.cr/educare

CORREO: educare@una.cr

Resumen: El presente artículo tiene como objetivo principal analizar los rasgos del sentido de vida, motivación y sentido de propósito, como los más influentes en la permanencia en los estudios de un grupo de madres adolescentes. Se trabajó desde un paradigma interpretativo, con un enfoque cualitativo y un método fenomenológico; la información se recopiló mediante el uso de técnicas como la entrevista en profundidad y grupos focales, aplicados a once madres adolescentes con edades entre los 14 y 17 años, estudiantes del sistema educativo formal diurno público; pertenecientes a tres instituciones urbanas ubicadas en la Gran Área Metropolitana en los meses de octubre y noviembre de 2015. Los resultados obtenidos permiten identificar los rasgos de sentido de vida: motivación al logro y sentido de propósito como factores determinantes para que estas jóvenes permanezcan estudiando, además se resalta la labor que desde la orientación se puede llevar a cabo con madres adolescentes.

Palabras claves: Orientación; madres adolescentes; motivación al logro; sentido de propósito; permanencia.

Abstract: This article derives from the research project titled "Features of the meaning of life that favor the permanence of teenage mothers in the formal education system". Its main objective was to analyze the features of the sense of life, motivation and a sense of purpose, as the most influential factors for the retention of teenage mothers in the formal education system. We worked from an interpretive paradigm, with a qualitative approach and phenomenological method. The information was collected by using techniques such as in-depth interviews and focus groups applied to eleven adolescent mothers aged between 14 and 17 years old; they were students in the formal public education system, day mode, from three urban institutions located in San Jose's Greater Metropolitan Area, Costa Rica. The research was conducted in the months of October and November, 2015. The results allow identifying the traits of the meaning of life: achievement motivation and a sense of purpose as the most decisive factors for these girls to remain in the high school; also the work and the contribution that the profession of counseling can promote is highlighted.

Keywords: Counseling; teenage mothers; achievement motivation; a sense of purpose; permanence.

Resumo: O principal objetivo deste artigo é analisar as características do sentido da vida, motivação e senso de propósito, como fatores que mais influenciam na permanência em estabelecimentos de ensino, de um grupo de mães adolescentes. $O$ trabalho foi realizado a partir de um paradigma interpretativo, com abordagem qualitativa e método fenomenológico; a informação foi coletada através da utilização de técnicas como entrevistas em profundidade e grupos focais, aplicados a onze mães adolescentes com idades entre 14 e 17 anos, alunas do diurno em instituições públicas de ensino formal; localizadas na Grande Área Metropolitana, nos meses de outubro e novembro de 2015. Os resultados permitem identificar ideias sobre o sentido da vida: motivação para a realização de trabalhos e senso de propósito como determinantes para essas jovens continuar estudando. Além disso, enfatiza-se o trabalho desde a orientação educativa, que pode ser realizado com as mães adolescentes.

Palavras-chave: Orientação; mães adolescentes; motivação a lograr metas; senso de propósito; permanência. 


\section{Introducción}

En Costa Rica, el número de adolescentes que quedan embarazadas en la etapa escolar ha aumentado en los últimos años, los datos ofrecidos por el Instituto Nacional de Estadística y Censos (INEC) revelan que para el 2012 la cantidad de mujeres embarazadas entre los 15 y 19 años fue de 13.672; en donde 522 casos corresponden a jóvenes menores de 15 años, aunado a esto, el $90 \%$ de las madres adolescentes deserta del sistema educativo, pues se les presenta la obligación de dividir su tiempo para el cuido de sus hijas o hijos, y muy poco para el estudio (INEC, 2013).

Aunado a lo anterior, el Departamento de Análisis Estadístico del Ministerio de Educación Pública de Costa Rica (MEP, 2015) reporta que, en la matrícula de estudiantes en el sistema educativo formal, durante el ciclo lectivo del año 2013, existe un promedio anual de 1146 estudiantes menores de edad en estado de embarazo y un promedio anual de 1.136 estudiantes menores de edad que son madres.

Algunas de estas adolescentes permanecen en las aulas, mientras que otras desertan de ellas por diversos motivos: falta de vínculo con el sistema educativo, problemas económicos, falta de apoyo por parte de sus personas cercanas, falta de sentido de propósito, entre otros. Por esta razón, investigar de qué manera los rasgos de sentido de vida repercuten como factores de permanencia escolar, además de determinar los rasgos que les permiten cumplir con su rol de madre y educanda, es relevante para una disciplina educativa como lo es la orientación, ya que esta busca promover el fortalecimiento de habilidades necesarias para que las personas enfrenten los diferentes retos que se presentan en su vida cotidiana.

De forma tal que, cuando se relaciona el sentido de vida con las madres adolescentes que continúan en el sistema educativo formal, surge la interrogante: ¿Cuáles rasgos del sentido de vida que presentan estas mujeres, resultan más significativos que los demás, en relación con su permanencia en el sistema educativo formal, aún con el cambio abrupto que ha dado su cotidianidad?

Las respuestas a esta pregunta pueden ser herramientas importantes para la labor orientadora, pues la orientación tiene un papel fundamental en las instituciones, ya que desde su visión educadora, se pueden fortalecer aspectos como la toma de decisiones y proyecto de vida en la población orientada, además de fortalecer los rasgos del sentido de vida como la motivación al logro y el sentido de propósito que, de acuerdo con lo descubierto en la investigación, se desprenden como aquellos que marcan una diferencia en la permanencia de las madres adolescentes dentro de los centros educativos.

\section{Fundamentación teórica}

Este apartado pretende realizar una aproximación teórica acerca del embarazo adolescente y el significado de ser madre en esta etapa, además de enfatizar en el sentido de vida, así como en los rasgos considerados factores de permanencia de las madres adolescentes en el sistema 
doi: http://dx.doi.org/10.15359/ree.21-3.8

URL: http://www.una.ac.cr/educare

CORREO: educare@una.cr

educativo formal diurno costarricense. También se presenta el rol de la persona profesional en orientación en la atención a la madre adolescente estudiante.

\section{Madre adolescente}

La adolescencia ha tenido una serie de variantes de acuerdo con el contexto y la cultura. Krauskopf-Roger (2011) se refiere a algunas transformaciones psicosociales de la adolescencia, entre las cuales se da una importante preocupación por la afirmación personal y social. Además, emergen durante esta etapa impulsos en el área sexual, por lo que se hace necesaria la promoción de conductas sexuales que denoten integralidad y responsabilidad, puesto que se incrementa en las adolescentes y los adolescentes el interés por experimentar el amor de una pareja.

Además, en esta etapa, la persona adolescente establece su punto de vista a raíz de las experiencias nuevas, pues le aportan madurez en distintas áreas como la sexual, psicológica, laboral, política y social (Donas-Burak, 2001; Fuller-Osores, 2001; Krauskopf-Roger, 1994); en donde el área sexual adquiere mayor importancia, pues se está en una condición que podría resultar riesgosa en caso de presentarse un embarazo.

Al respecto, Andrade-Ruízy Sánchez-Solano (2000) mencionan que, aunque la adolescente se encuentra preparada genitalmente para la reproducción sexual, desde lo sociocultural no está lista para asumir con responsabilidad el producto de dicha acción. No obstante, la forma en que se asuma esta situación puede variar según cada persona, su historia de vida y el contexto en el que se desenvuelve, aun cuando esta se encuentre en la etapa de la adolescencia.

Por su parte, autores como Coll (2001) e Issler (2001) señalan que la actitud que las adolescentes adopten frente a esta circunstancia va a depender, en gran parte, de la etapa de la adolescencia que están experimentando, el significado que su hija o hijo tenga para ella, el origen del embarazo, su historia de vida, contexto familiar y social, su proyecto de vida antes de embarazarse. Tal actitud puede indicar asimilación del proceso, mas no preparación.

En este sentido, la maternidad representa un desafío, pues limita el alcance de metas, en donde el posible rechazo inicial de la familia, la pareja, la escuela y la sociedad es otra situación que podría afectar notablemente la vida de dichas jóvenes, y provocar profundos efectos en el desarrollo y bienestar de las adolescentes, pues el sentido psicológico del embarazo se forma a partir de la prevalencia de experiencias negativas, como sentimientos de culpa, vergüenza, sensación de aturdimiento y extrañeza (Caballero-Batista y Méndez-Díaz, 2005; Nava-Flores, 2009).

Aunado a lo anterior, se destaca la ruptura biográfica en la cotidianidad y modo de vida de las madres adolescentes, quienes deben asumir un nuevo rol (Celedón y Garri, 2014; Quintero-Rondón y Rojas-Betancur, 2015), lo que las lleva a replantear eventualmente, un nuevo proyecto de vida que esté centrado en su hija o hijo, afectando directamente las proyecciones y metas de esta población, las que se encuentran completamente vinculadas con la construcción de su sentido de la vida.

4 


\section{Sentido de vida en madres adolescentes}

Las adolescentes no tienen un sentido de vida definido, sino que se aproximan a reconocer las respuestas que componen su significado y los rasgos que lo expresan; la principal fuerza que motiva a la persona es la intencionalidad y la lucha por encontrarle un sentido a su propia vida, manifiesta esta, de manera dinámica y continua, enmarcada por una serie de vivencias a nivel social, amoroso y familliar (Frankl, 1991; Lukas, 2003; Ortiz-Arias, 2012).

Así, la persona debe trabajar activamente para definir cuál es su sentido de vida, ya que este no llega de repente y sin un análisis intencionado y dirigido. Por lo que se puede afirmar que la madre adolescente no debería esperar a que la vida le dé su sentido, sino ella misma ir encontrando su significado, e involucrar lo que este conlleva: libertad, voluntad y responsabilidad.

En consecuencia, la madre adolescente posee voluntad de sentido que, según Frankl (1991), corresponde a la búsqueda del sentido que hace la persona a lo largo de su vida, movida por una fuerza de instinto, primaria y vital. Es único, subjetivo, específico y relativo en cuanto a lo que es cada quien y el contexto en el que se encuentra, por lo que también cada quien se hace responsable de buscarlo y hallarlo; satisfaciendo esa voluntad (Frankl, 1991; Loaiza-Valdés, 2005; Noblejas-De la Flor, 2000).

Por lo anterior, se puede considerar fundamental para la madre adolescente el poseer y clarificar los aspectos de su sentido de vida, lo que otorga significado a las situaciones y circunstancias del sumario vital que se le presenten. De igual forma, dentro del sentido de vida se ubican los rasgos de este mismo, que son esas características que juntas dan lugar a su expresión, estos se mantienen entrelazados y en constante interacción unos con otros.

\section{Rasgos del sentido de vida como factores de permanencia}

De acuerdo con Jiménez-Segura y Arguedas-Negrini (2004), la construcción del sentido de vida se basa en algunos pilares fundamentales, definidos como rasgos del sentido de vida, los cuales son elementos que las madres adolescentes tienden a manifestar a medida que elaboran y fortalecen su significado trascendental de vida. A su vez, un factor de permanencia o factor de protección es aquella característica o recurso vinculado con patrones conductuales y estilos de vida, que se adquiere y fortalece en la interacción con elementos ambientales como la escuela y la familia, para favorecer la continuidad de cada estudiante en el sistema educativo (Arguedas-Negrini, 2010; Secretaría de Seguridad Pública de México, Gobierno Federal de los Estados Unidos Mexicanos, 2011).

En función de este concepto, se resaltan tres puntos, estos factores al ser características, se asocian con rasgos propios de cada madre adolescente, para este artículo se toman en cuenta dos: la motivación al logro y el sentido de propósito. Segundo, estos se encuentran en constante 
doi: http://dx.doi.org/10.15359/ree.21-3.8

URL: http://www.una.ac.cr/educare

CORREO: educare@una.cr

dinámica, se impactan unos a otros, lo que imposibilita su segregación, así pueden concebirse como una red que potencia y fomenta que estas jóvenes continúen formándose en el ámbito educativo; y tercero, se hallan en contextos más próximos a la muchacha y son sistemas de impacto como el familiar y la institución educativa, en los que se pueden aprender, adquirir y potenciar.

Ahora bien, Masten y Reed (citados por Wong y Wong, 2011) indican que los rasgos funcionan como factores protectores, en las situaciones difíciles o complejas; por tanto, al considerar como referencia ambas propuestas, se señalan ocho rasgos como factores adecuados a las madres adolescentes: fe y espiritualidad, aspiraciones educativas, metas, intereses especiales, optimismo, persistencia, motivación al logro y sentido de propósito; de estos, se destacan los dos últimos para el interés del presente escrito, pues se asocian más con la permanencia de las jóvenes en el sistema educativo.

Motivación al logro: se define como una fuerza interna que lleva a las personas a esforzarse por mejorar o satisfacer un determinado criterio de excelencia; lo que se refleja en las acciones concretas realizadas para alcanzar las satisfacciones que buscan. Tal ímpetu está relacionado con el concepto de la autorrealización que define esta motivación como un proceso de planteamiento y esfuerzo por realizar las tareas de la mejor manera posible (Jiménez-Segura y Arguedas-Negrini, 2004; Paz-López, 2005). De ahí que la motivación al logro sea vista como un impulso que adquiere la madre adolescente para realizar diversas tareas en su vida cotidiana; $y$ en estas es fundamental llevar a cabo labores que deben asumir en el accionar de su nuevo rol, sin dejar de lado los que les eran previos.

Igualmente, Beltrán-Llera y Bueno-Álvarez (1995) y Jiménez-Segura y Arguedas-Negrini (2004) mencionan que esta motivación se dirige al éxito y cuando la persona valora que el motivo para lograr una meta es mayor al temor de no alcanzarla, está más motivada hacia el logro que al fracaso. Así, la motivación al logro es un impulso interno de la madre adolescente, para lograr un objetivo concreto con éxito, establecido ante una necesidad y que contribuye a su satisfacción personal; en donde esa orientación hacia un algo puede hallarse dentro del sentido de propósito que le confiere.

Sentido de propósito: el sentido de propósito se encuentra estrechamente vinculado con los aportes que realizó Frankl (1991) sobre la noción de voluntad de sentido. Este destacó que la búsqueda que hacen las personas de un sentido para su vida es una acción primaria de los impulsos instintivos y no una "racionalización de tipo secundaria". En virtud de estos señalamientos, Jiménez-Segura y Arguedas-Negrini (2004) definen este rasgo del sentido de la vida como aquella intención o visión propia de cada persona sobre su futuro, caracterizada por un impulso vital, con la que esta se ha identificado a elección individual y que, por ende, le genera satisfacción. 
Se concibe, entonces, como una fuerza que mueve a la joven madre hacia la dirección con la que ha encontrado una identificación en correspondencia con sus ideales de mundo y proyecciones de futuro, determinando su trazado en el cumplimiento de las metas que la motivan a continuar transitando su trayecto vital.

A raíz de lo anterior, se plantea que el sentido de vida puede ser construido a lo largo del ciclo vital y una de las tareas que se puede ejecutar desde la orientación es, precisamente, fortalecer esos rasgos para que la persona y, en concreto, la madre adolescente pueda alcanzar altos niveles de satisfacción personal e impacto positivo en las diferentes áreas de su vida, una de ellas la educativa; por lo tanto, la orientación debe responder a las necesidades de dicha población, de acuerdo con las características que se mencionan enseguida.

\section{Papel de la persona profesional en orientación en la atención a la madre adolescente estudiante}

La orientación es una disciplina aplicada que, por sus características y por su objeto de estudio, se entiende como un proceso permanente de interacción, que se da a lo largo de toda la vida del sujeto en correspondencia con su entorno. De tal forma, esta profesión es concebida como parte integrante del proceso y proyecto educativo, que define como su sujeto de estudio a la persona, considerada un sistema integral, en donde el objeto de estudio son los diferentes procesos que forman parte del proyecto que la persona orientada emprende a lo largo de su vida: estos se encuentran completamente interrelacionados y dinamizados entre sí.

Por ello, para que la atención e intervención en la situación de embarazo adolescente se realice de la mejor manera, se proponen tres aspectos fundamentales para satisfacer las necesidades que lasjóvenes presentan en ese momento y, además, para promover su permanencia en el sistema educativo (Cartin, 2011). El primero es la intervención básica o de primer orden, que pretende salvaguardar los derechos de la madre adolescente, en donde la persona profesional en orientación deberá escuchar las necesidades de la estudiante, sin desvalorizar sus sentimientos; utilizar un lenguaje agradable brindando apoyo dentro de lo posible y acompañar y coordinar con otras entidades, así como dar asesoramiento de las adecuaciones de horarios.

El segundo es el apoyo educativo, del cual dependerá, en gran medida, la permanencia de la adolescente en la institución, en este caso corresponde aplicar de manera temporal una adecuación curricular; justificar ausencias; dar un seguimiento mediante trabajos extra clase; permiso por lactancia materna (1 hora diaria); hacer ajustes en el procedimiento para la calificación del trabajo cotidiano, asistencia y aplicación de pruebas; proveer a la adolescente mobiliario adecuado; y brindar información y apoyo sobre las posibles alternativas de cuido. Si la adolescente se retira del sistema educativo, debe dársele seguimiento para verificar la situación real por la cual se retira y buscar conjuntamente alternativas para promover la reinserción al año siguiente. 
doi: http://dx.doi.org/10.15359/ree.21-3.8

URL: http://www.una.ac.cr/educare

CORREO: educare@una.cr

El tercer aspecto representa el apoyo interinstitucional que requiere la articulación y coordinación entre el Ministerio de Educación Pública (MEP) y las instituciones vinculadas con la atención de las niñas y adolescentes embarazadas entre ellas: Fondo Nacional de Becas (FONABE), Patronato Nacional de la Infancia (PANI), Instituto Mixto de Ayuda Social (IMAS), Instituto Nacional de las Mujeres (INAMU), Caja Costarricense de Seguro Social (CCSS), Instituto Nacional de Aprendizaje (INA).

Todos los aspectos mencionados permiten realizar una intervención desde la orientación de una forma más efectiva, en procura del bienestar de la madre adolescente, por lo que es menester conocer las directrices, las leyes y todos los aspectos que resguardan a esta población dentro del sistema educativo, para promover su permanencia en garantía de su desarrollo pleno y el de su hija o hijo.

\section{Métodología}

La investigación de la cual se desprende el presente artículo se ubica en el paradigma naturalista, también llamado interpretativo, ya que su interés radica en el estudio de los significados de las acciones humanas y la vida social (Barrantes-Echeverría, 2003; GurdiánFernández, 2007; Hernández-Sampieri, Fernández-Collado y Baptista-Lucio, 2003). A su vez, lo naturalista o interpretativo contempla el enfoque cualitativo, que permite tener acceso a la realidad de los sujetos de la investigación, pues se parte de sus vivencias y experiencias y se toma en consideración la diversidad de significados que los mismos aportan.

Se optó por el método fenomenológico, ya que este, hace posible la interpretación de los procesos sociales, estudiando las realidades desde la propia perspectiva de la persona, es decir, examina la forma en la que se experimenta el mundo, en donde lo fundamental es lo que las personas perciben significativo de sus experiencias (Rojas-Valenciano, 2008; Taylor y Bogdan, 1987). De esta forma, el método fenomenológico permitió un acercamiento directo con las once madres adolescentes con edades entre los 14 y 17 años, que asisten al sistema educativo diurno formal de Costa Rica, así como también a su realidad, en su rol de madre, adolescente y estudiante.

Los tres centros educativos diurnos a los que asisten las madres adolescentes que participaron de esta investigación se ubican en la Gran Área Metropolitana de la provincia de San José; estas instituciones han sido contempladas puesto que proporcionan un número elevado de estudiantes adolescentes madres.

El visto bueno para la entrada a las instituciones fue solicitado inicialmente vía telefónica y en segunda instancia mediante una carta, posteriormente, se procedió a realizar el primer acercamiento con los Departamentos de Orientación y las estudiantes madres adolescentes, para concertar las entrevistas que se llevaron a cabo tomando en consideración el uso del consentimiento informado que fue firmado por las estudiantes madres y las madres de estas mismas.

8 
Con la finalidad de obtener un acercamiento directo a las participantes, así como a la descripción de sus vivencias, se seleccionó la técnica de entrevista a profundidad, para lograr identificar aquellos rasgos de sentido de vida que favorecen la permanencia de las jóvenes madres en el sistema educativo diurno formal de Costa Rica. Además, se triangula la información usando grupos focales con las madres adolescentes y con el personal de orientación.

Posterior a la recolección de los datos, se procedió a analizar e interpretarlos mediante la elaboración de categorías de análisis. Para el procesamiento y análisis de la información se utilizó el diseño de Taylor y Bogdan (1987), el cual consta de tres fases: la primera de ellas, descubrimiento en progreso, buscó identificar temas emergentes, conceptos y proposiciones que dieran sentido a los datos. Para llevarla a cabo, fue necesario contar con las entrevistas y grupos focales transcritos, pues ello permitió identificar y relacionar la información obtenida con la teoría. La segunda fase, codificación, incluyó la codificación de la información y la reunión de toda la información referente a temas, ideas, conceptos, interpretaciones y proposiciones. Una vez codificado el material, se elaboraron las categorías y subcategorías de análisis. La tercera fase, relativización de los datos, implicó su análisis e interpretación, así como la elaboración y revisión de conclusiones, mediante el apoyo teórico y la información recopilada. Finalmente, se elabora el informe de investigación, del cual se desprende el presente artículo.

\section{Análisis de los resultados}

A partir de los datos recolectados de las diversas fuentes utilizadas: experiencias de las jóvenes madres y personal de orientación; se presentan categorías y subcategorías de análisis para interpretar la información recopilada en congruencia con la teoría. Así, de los ocho rasgos del sentido de vida: intereses especiales, metas, motivación al logro, aspiraciones educativas, optimismo, persistencia, fe y espiritualidad y sentido de propósito, se destacan dos como los más influyentes en la permanencia de las jóvenes madres en el sistema educativo, estos son precisamente la motivación al logro y el sentido de propósito, por tal razón se ahonda más en ambos rasgos.

\section{Motivación para el logro académico}

Para las madres adolescentes entrevistadas, la motivación al logro implica identificarse o mantenerse con voluntad de conseguir algo previamente determinado, es decir, un anhelo por hacer realidad un determinado deseo, sea este algo material o inmaterial. En este sentido, Beltrán-Llera y Bueno-Álvarez (1995), Jiménez-Segura y Arguedas-Negrini (2004) afirman que una persona motivada al logro valora más la posibilidad de conseguir el objetivo, que el temor de no de hacerlo. Se despliegan tres sub-categorías en las que se distinguen las acciones necesarias para la obtención y el reconocimiento de los logros académicos, así como los motivos significativos para ellos. 
doi: http://dx.doi.org/10.15359/ree.21-3.8

URL: http://www.una.ac.cr/educare

CORREO: educare@una.cr

\section{- Acciones para alcanzar logros académicos}

Se conciben como aquellas tareas cotidianas que la joven estudiante realiza para alcanzar metas académicas con éxito. De acuerdo con las afirmaciones de las entrevistadas a partir del momento en que empiezan a combinar las responsabilidades como madres adolescentes y estudiantes, se marca un cambio en las tareas cotidianas que llevaban a cabo, tales como cumplir con trabajos académicos o estudiar para exámenes.

Para obtener algo ... se me hacía muy fácil, por que solo se lo pedía a mis papás y me lo daban, ... no tenía que hacer mucho esfuerzo. Entrevistada 1.

... solo me importaba estar con mis amigas o no pensaba ni en el estudio y ahora yo sé que ya tengo una hija, y tengo que darle lo mejor, ... me da fuerzas para venir al colegio. Entrevistada 8.

Esforzarme para salir bien, hacer caso en todo lo que me digan, con respecto al colegio, cumplir con todas mis obligaciones. Entrevistada 7.

Estudiar bastante, ... no puedo estudiar como que mucho pero ahí lo que puedo estudiar, cuando la bebé se duerme o como así, así logro estudiar. Entrevistada 11.

Estas jóvenes describen que previo a los embarazos, su cotidianidad tendía a ser más libre en el sentido de que tenían mínimas obligaciones, algunas de las cuales incluso podían decidir si llevar a cabo o no, por ejemplo, era posible elegir ausentarse del colegio sin mayores consecuencias; sin embargo, esto es muy diferente a partir de que son madres, ya que reconocen la importancia de ser responsables en su proceso de aprendizaje para aprobar el nivel que cursan.

Desde el punto de vista sociocultural, una adolescente no se encuentra preparada para asumir las responsabilidades provenientes de la procreación y educación de un hijo o una hija (Andrade-Ruíz y Sánchez-Solano, 2000), ya que se contraponen a las obligaciones propias de esta etapa del desarrollo, enfocadas en gran parte en lo académico.

Aunado a lo anterior, para alcanzar el éxito escolar las madres adolescentes necesitan priorizar sus tareas, organizándolas de manera tal que sus quehaceres como madres tienen la mayor importancia en su vida, seguidas muy de cerca por las responsabilidades académicas. Es decir, se evidencia cómo los deberes escolares pueden cumplirse una vez que sus funciones como madres han sido completadas, reflejando una alta motivación para lograr las tareas de su rol como madre y estudiante.

De forma tal, que una de las principales acciones para alcanzar los logros académicos hace referencia a la organización del tiempo de la madre en función de los cuidados de su hija o hijo, por 
ejemplo, estudiar hasta el momento en que su bebé se ha dormido o levantarse temprano para llevar a cabo sus tareas cotidianas. Así, estas jóvenes hacen grandes esfuerzos para desenvolverse en ambos roles, sin embargo, antes de tener a sus hijas e hijos ellas no aplicaban tanto esfuerzo en sus estudios, ya que declaran que no les parecía algo trascendental en sus vidas, por lo tanto, después del embarazo se aprecia un incremento en la responsabilidad académica.

Consecuentemente se puede afirmar que, en el ejercicio de la maternidad adolescente surgen diversas necesidades que deben ser satisfechas, por lo cual, a través de las acciones y los motivos significativos para el logro, estas madres pueden alcanzar las metas propuestas para su satisfacción personal y académica.

\section{- Motivos significativos para el logro académico}

En particular, los motivos significativos para el logro académico son razones de importancia para la madre adolescente que motivan su permanencia en el sistema educativo. En las entrevistas realizadas se destaca que la mayor parte de las respuestas se enfocan en que el motivo más significativo para el logro académico es el bienestar de sus hijas o hijos. Esto se extiende a los anhelos y pretensiones que la joven madre expresa, dirigidos a conseguir una mejor calidad de vida.

Salir adelante con mi hijo, y darle un buen futuro a él... . Yo necesito salir por mí misma adelante, ... sacar al bebé adelante y no necesitar de nadie. Entrevistada 1.

A mí lo que me motiva es ver a mi bebé y ver que yo por él voy a salir adelante, él es como mi motorcito que me está ayudando para poder salir. Entrevistada 4.

Di mi bebé, la verdad, porque ... no quiero que ella salga igual que mi ... darle un mejor futuro a ella ... sí ... para que ella también estudie. Entrevistada 10.

A partir de la maternidad, el proceso educativo adquiere un nuevo significado para las adolescentes en términos de valoración, pues hay una claridad en la importancia de lo que representa continuar esforzándose para adquirir logros académicos, puesto que ello favorecerá su crecimiento personal y el bienestar de su bebé. Esta motivación hace que las acciones realizadas sean constantes y direccionadas para alcanzar sus metas, explican el comportamiento que asumen las adolescentes ante su rol de madres y estudiantes, tomando en cuenta el contexto sociocultural en el que están, de tal forma que desean salir adelante a través de sus propios esfuerzos (Santrock, 2002). 
doi: http://dx.doi.org/10.15359/ree.21-3.8

URL: http://www.una.ac.cr/educare

CORREO: educare@una.cr

Un aspecto por resaltar es que las madres adolescentes participantes de esta investigación se encuentran inmersas en contextos vulnerables, en donde se manifiestan condiciones de pobreza, baja escolaridad de familiares y hacinamiento; a pesar de estas condiciones, el ser madre para estas jóvenes se convierte en un impulso de búsqueda de nuevas oportunidades mediante el estudio.

Además de los propios recursos, las madres adolescentes requieren de redes de apoyo que se encuentran en los servicios ofrecidos por las instituciones educativas, en lineamientos previamente establecidos por el MEP, dado que esto les permite mayores oportunidades de movilidad social. Así lo afirma Espada-García (2006), quien asegura que, en ocasiones, además de la motivación propia, es necesario el apoyo externo, tal como lo es la educación y el reconocimiento propio y de otras personas de los logros académicos que las madres adolescentes alcanzan, ya que esto afirma su motivación para continuar estudiando.

\section{- Reconocimiento de logros académicos}

Se refiere a la validación propia y de otras personas a la madre adolescente, cuando alcanza un logro académico, la cual promueve su permanencia en el sistema educativo. Gracias a la información obtenida en las entrevistas se hace evidente que existen dos panoramas en cuanto al reconocimiento de los logros académicos en las madres adolescentes; uno de los cuales implica el reconocimiento que ellas se hacen a sí mismas y el que reciben de terceras personas, este último positivo en ocasiones y negativo o inexistente en otras.

Siempre tengo el apoyo de mi mamá, y mi papá, mi papá me motiva. Entrevistada 2.

Casi nadie ... ve cuando uno, ... hace las cosas bien; cuando uno hace las cosas mal todo mundo las ve..., entonces, sí se complica de cierta manera. Entrevistada 3.

Nadie. Entrevistada 4.

Feliz, contenta, como el año pasado que gané noveno porque yo pensaba que no iba a pasar porque había faltado como dos meses por el embarazo. Entrevistada 10.

Reconocer el logro académico de las madres adolescentes y su esfuerzo para superar las adversidades cotidianas de sus roles las alienta a sentirse seguras y realizadas con respecto a sus metas académicas. No obstante, no todas las madres adolescentes reciben dicho reconocimiento. Es necesario para las estudiantes recibir algún tipo de recompensa cuando tienen éxito en lo educativo y personal, tomando en cuenta sus habilidades y posibilidades de acuerdo con el contexto en el que se desenvuelven, pues lo anterior sirve como base para la autorrealización. Este reconocimiento del logro académico fomenta la valoración personal de las madres adolescentes y fortalece su sentido de vida (Educarchile, 2014). 
Se debe agregar que esta compensación emocional al esfuerzo fomenta la permanencia de las jóvenes madres en el sistema educativo, a partir del incremento de la confianza en sí mismas y el sentido de responsabilidad en pro de los logros académicos. Esto presenta el vínculo entre el esfuerzo de la madre adolescente, el reconocimiento de sus logros y la construcción de su sentido de vida, lo que da dirección y sentido a sus acciones cotidianas para comportarse de una manera congruente con su nueva visión de mundo.

\section{- Sentido de propósito de la madre adolescente estudiante}

Se entiende como aquella experiencia introspectiva, en la que cada madre adolescente gradualmente ubica la respuesta al para qué de su vida. Significa la fuerza que determina su dirección en el cumplimiento de los ideales educativos y proyecciones de futuro. De tal forma que favorece la estabilidad de la joven estudiante en el logro de metas y en el fortalecimiento de su sentido de vida, lo que promueve su permanencia en el sistema educativo, así como su crecimiento personal. A partir de esta categoría surgen dos subcategorías, en las cuales se amplía acerca de las razones que aportan sentido a las vidas de las madres adolescentes y lo que para ellas significa la maternidad.

\section{- Razones que dan sentido a la vida de la madre adolescente estudiante}

Hacen referencia al significado que le da la adolescente a su nueva vida como madre, a su hija o hijo como la inspiración y el impulso para continuar y cumplir sus metas y deseos académicos, ya que se convierten en el principal motivo para enfrentar las situaciones que se le presentan en su vida diaria. Estos otorgan esperanza a la joven para seguir adelante. A raíz de la información recolectada, se muestra cómo este proceso de maternidad ha ayudado a la adolescente a fortalecer la construcción de su sentido de propósito, enfocándolo en su hija o hijo y sus estudios.

Mis bebés más que todo ... mi razón de vivir son ellos ahora. Entrevistada 2.

Cuando ya no puedo más y digo es que el bebé, es parte de mí, le da significado a mi vida, si yo no hubiera tenido a mi hijo, seguro sería la misma persona perdida de antes. Entrevistada 4.

Mi bebe y mi estudio. Entrevistada 6.

Mi bebé no es un estorbo y es la que me hace que yo siga en el colegio y que mis papás se sientan orgullosos de mí. Entrevistada 7. 
doi: http://dx.doi.org/10.15359/ree.21-3.8

URL: http://www.una.ac.cr/educare

CORREO: educare@una.cr

Un aspecto importante en cuanto al sentido de propósito de las madres adolescentes es que se despierta un mayor interés por el bienestar de sus hijas e hijos, así como el de ellas, lo que le da un valor diferente a sus vidas de acuerdo con su propia perspectiva, lo cual les ayuda a creer en sí mismas y les posibilita el enfrentar de forma positiva sus retos. Así, a partir de los cambios que las adolescentes experimentan en su proceso de maternidad, mientras se mantienen dentro del sistema educativo, se evidencia cómo estas obtienen una mayor claridad acerca de sus propias capacidades y habilidades, lo que se convierte en un factor protector que favorece la permanencia de dichas jóvenes en el sistema educativo.

Por ende, el convertirse en madres adolescentes, para estas jóvenes, lejos de significar un impedimento, resulta en un impulso que les ayuda a definir un "para qué" en sus vidas, lo que les motiva a combinar de manera positiva el rol de estudiante y madre, lo cual es causa de orgullo para algunas de ellas y para sus familiares, ya que se fortalece el reconocimiento de sus logros académicos y personales.

En relación con lo anterior, Cavallé (2013) se refiere a que el sentido de propósito envuelve el entendimiento acerca de la dirección y proveniencia de la propia vida, que en este caso se direcciona hacia las hijas o los hijos y de esta forma al poder dar respuesta a estas variables, las madres adolescentes obtienen seguridad y confianza, lo que les dispone para cumplir con la finalidad específica de proveer económica y emocionalmente a sus bebés.

En consecuencia, el hecho de ser madres adolescentes y enfrentarse a su realidad provoca que ellas puedan concebir a su hijas o hijos como la principal razón que da sentido a su vida, lo que favorece que las jóvenes persistan y continúen con sus estudios, pues su fin primordial es brindar estabilidad en pro del bienestar próximo y futuro tanto de sí mismas como para sus niñas o niños.

Asimismo, Frankl (1991) menciona que las personas conciben la búsqueda de su sentido de vida como una acción que refleja sus impulsos primarios y no como la racionalización de estos mismos; esto se concibe como sentido de propósito o voluntad de sentido, lo cual puede observarse en lo descrito anteriormente por las jóvenes quienes se centran en garantizar las mejores condiciones para sus hijas e hijos. A la vez, esa búsqueda de sentido se vincula estrechamente con el significado que las jóvenes le otorgan a su proceso de maternidad, lo que favorece la permanencia de las madres adolescentes en el sistema educativo costarricense.

\section{- Significado de la maternidad}

Cada joven estudiante otorga un significado al hecho de ser madre, dentro de lo cual se conjugan los sentimientos y las emociones experimentadas, así como el aprendizaje y la responsabilidad que conlleva dicha vivencia para esta población. De acuerdo con lo expresado por las jóvenes, se destaca el significado de la maternidad tomando en cuenta tanto el disfrute de ello como el trabajo y dificultades que esta implica en la vida diaria a nivel personal y escolar. 
He renunciado ... a mi libertad... como soy una güila me quisiera como arreglar más, ..., he renunciado como a mi adolescencia.... Entrevistada 1.

... la maternidad ha sido, un cambio, radical, de forma, tanto psicológica como física ... es, ... algo muy bonito, es ya tener un hijo para toda la vida, un acompañante, ... a pesar de todo, a pesar las circunstancias yo digo que el amor de un hijo es el que nunca se acaba. Entrevistada 3.

Es algo precioso que no se puede describir, aunque a veces cansa, ... pero es lo mejor, aunque canse, aunque joda, ..., pero no cambiaría eso por nada. Entrevistada 4.

... una responsabilidad muy grande pero no imposible de lograr, ..., significa dar todo por esa persona, demostrar que uno no puede caer porque hay alguien que sigue los pasos de uno, que uno no puede ser débil... . Entrevistada 8.

Uno de los principales significados que las adolescentes dan a su maternidad se relaciona con las modificaciones que han tenido que realizar en su estilo de vida, en cuanto a actividades y preferencias propias de su etapa de desarrollo, así como en su cotidianidad, tales como dejar de maquillarse, hacerse más responsables y estar a cargo del cuido de sus bebés. De igual forma, se aprecia cómo, desde la perspectiva de estas jóvenes, el hecho de haber quedado embarazadas implica asumir un compromiso que se considera como una consecuencia negativa de sus comportamientos pasados, pues, se ve como una carga adquirida por una conducta socialmente inadecuada para su etapa de desarrollo, como lo es, mantener relaciones sexuales sin protección y a corta edad, obligándoles a tomar las responsabilidades de ser madres.

En relación con lo anterior, se percibe una renuncia a la libertad y una pérdida de su adolescencia, por lo que la maternidad representa un cambio trascendental. Se destaca lo psicológico, como el asumir cambios en cuanto a responsabilidades, retos enfrentados y el hecho de que sus actividades sociales se encuentran supeditadas al cuidado de sus hijas e hijos y a la presión que ellas perciben para no mostrar debilidad ni equivocarse buscando ser un buen ejemplo para sus bebés.

No obstante, a pesar de las dificultades y el cansancio que implica el ser madres, las jóvenes aluden también a que gracias a la maternidad experimentan sentimientos y actitudes positivas tales como amor incondicional, alegría, compañía, entrega, fortaleza, esfuerzo, dedicación y paciencia. Consecuentemente, tales sentimientos y actitudes direccionados hacia sus hijas e hijos forman parte también del significado que estas jóvenes dan a la maternidad y que a la vez se convierte en una fuerza interna que promueve su continuidad en los estudios.

Estas adolescentes visualizan el ser madres como algo irrenunciable en términos de que su hija o hijo es quien le acompañará durante toda su vida y que no cambiarían por nada, tal y como lo 
doi: http://dx.doi.org/10.15359/ree.21-3.8

URL: http://www.una.ac.cr/educare

CORREO: educare@una.cr

menciona Coll (2001), al indicar que el niño o la niña se concibe como algo propio, lo que haciendo que se sientan totalmente responsables de esta nueva persona. Al respecto, Quintero-Rondón y Rojas-Betancur (2015) indican algunos cambios en la cotidianidad de las madres adolescentes, tales como la aceptación de su maternidad, lo que las lleva a realizar los ajustes necesarios para enfrentar su rol de estudiantes y el nuevo rol de madre, que se asume de manera resignada.

Esto se evidencia en la toma de decisiones a nivel académico y personal que lleva a cabo la madre adolescente cuando antepone el bienestar y cuido de su hija o hijo ante sus intereses o gustos personales, ya que se sacrifican en responder a las necesidades de sus bebés como su prioridad.

Es así que este proceso de maternidad es único y específico en cada una de las madres adolescentes, pues se encuentra determinado por su experiencia de vida así como por el contexto en el que se desenvuelven, lo que se relaciona estrechamente con la búsqueda del sentido de vida por parte de las jóvenes, que determina su accionar en pro de satisfacer su voluntad, lo que resulta ser un factor protector que promueve la permanencia de la adolescente en el sistema educativo (Frankl, 1991; Noblejas-De la Flor, 2000).

Finalmente, es importante retomar la relevancia de estos rasgos del sentido de vida como promotores de la permanencia de la madre adolescente en el sistema educativo, ya que ellas, impulsadas por la motivación del bienestar de su hija o hijo, así como el propio, logran formular metas que marcan las razones para construir su sentido de propósito. Ello es pertinente a la labor orientadora para el planteamiento de procesos de orientación a nivel individual y grupal, de forma tal que se atiendan las necesidades presentadas por las estudiantes al asumir el proceso de maternidad, con el fin de lograr su permanencia en las instituciones educativas.

\section{La orientación y el trabajo con madres adolescentes que estudian en el sistema educativo costarricense}

Según las madres adolescentes partícipes de la investigación, el servicio de orientación es concebido como el acompañamiento y asesoramiento que las adolescentes han recibido en su proceso de maternidad por parte del Departamento de Orientación correspondiente al centro educativo en el que se encuentran; aunado a las funciones, tareas o expectativas de lo que la persona profesional en el área debe realizar, en función de las necesidades específicas de esta población.

Algunas de las madres adolescentes aducen que a partir del apoyo individual recibido por el servicio de orientación han logrado obtener más confianza, seguridad en sí mismas y ciertas herramientas para afrontar situaciones cotidianas, ya que la persona profesional consigue establecer una relación de confianza con las estudiantes, lo que les permite conocer y conversar sobre las necesidades que estas jóvenes presentan. 
Se destaca, según la información recolectada, que el principal apoyo de la persona profesional en orientación a la estudiante, en su proceso de maternidad, consiste en informar y en los casos en que es necesario, tramitar el apoyo económico al que pueden acceder las madres adolescentes, por ejemplo, becas.

En cuanto a las funciones de la orientación educativa, estas son atinentes principalmente al desempeño y éxito escolar de sus estudiantes y la situación de maternidad no excluye a estas alumnas de esos fines primordiales, por cuanto se busca que ellas permanezcan en el sector educativo, logren sus metas en este ámbito y se desarrollen en todas las áreas de su vida de forma satisfactoria.

En consecuencia, la orientación debe atender tres principios en los cuales subyace: prevención, desarrollo e intervención social. La primera tarea a cumplir es anticipar conductas de riesgo que generen como consecuencia el embarazo adolescente, en otras palabras, prevención primaria, ya que la maternidad no es parte de las tareas propias de la etapa de la adolescencia.

Asimismo, en las entrevistas realizadas se evidencian tareas asociadas a la intervención social. No obstante, para las madres adolescentes que se encuentran en centros educativos y están situaciones de vulnerabilidad, y que se enfrentan al reto de asumir su rol de madre y estudiante de forma paralela; la atención de las orientadoras y los orientadores puede darse partiendo de la prevención secundaria, la cual está en función de que la estudiante y madre no deserte del sistema educativo.

Para ello es fundamental que la persona profesional de orientación planifique y ejecute acciones concretas que promuevan la permanencia de esta población y la promoción y fortalecimiento de los rasgos del sentido de vida se convierta en un aspecto que proteja a la joven y la prevenga de abandonar sus estudios. En esto, la construcción del sentido de vida puede verse favorecida por la atención grupal e individual con la finalidad de propiciar en las madres adolescentes el conocimiento de sí mismas, la clarificación de su identidad, el conocimiento del medio y la toma de decisiones, estas intervenciones son relevantes para la atención de tal población y su continuidad dentro del sistema educativo.

Así, en un primer sentido, es fundamental trabajar un proceso dirigido al autoconocimiento en todas sus generalidades, pero específicamente respecto a los rasgos del sentido de vida atinentes a los intereses especiales, los valores y las creencias personales, para que el estudiantado sea consciente de cuáles son sus pasatiempos, en qué actividades invertir su espacio de ocio y la forma en la que esto influye en la organización personal de su tiempo.

Además, es trascendental que esta población reconozca qué es lo más importante en su vida, lo que le da sustento, lo que le caracteriza. Otro de los aspectos que se deben retomar es el motivo más importante para conseguir esas metas, el impulso interno o externo, con el fin de responder a la motivación para el logro. El estudiantado debe ser consciente de cuál es su motivación para el logro, pues este nutre la consecución y formulación de sus metas. 
doi: http://dx.doi.org/10.15359/ree.21-3.8

URL: http://www.una.ac.cr/educare

CORREO: educare@una.cr

De igual forma, al trabajar las metas, se puede enfatizar en las aspiraciones educativas, como aquellas proyecciones que tienen que ver estrictamente con el área educativa y la fuerte influencia de estas en la permanencia en el colegio. También, se deben propiciar espacios para que las orientadas, como parte de su conocimiento del medio, reconozcan sus redes de apoyo, el tipo de soporte ya sea emocional o económico, que estas les brindan y su impacto en el funcionamiento de su permanencia en el sistema educativo.

Ligado a lo anterior, durante el embarazo la toma de decisiones viene a ser un proceso valioso para la adolescente en cuanto a la permanencia en el colegio, ligado a la temática de las metas a corto, mediano, o bien, largo plazo, las cuales pueden responder a aspiraciones educativas y funcionar como parte de la motivación al logro de la joven.

Es así cómo, en el durante y después del embarazo, la madre adolescente estudiante puede ubicar como cambio importante, tener un hijo o una hija, además de las transformaciones generales que se han dado a partir de esa vivencia y las características personales o sociales que se mantienen y a las que ella les da importancia. El orientador o la orientadora, en esa guía de reflexión a la joven estudiante, debe favorecer la integración de todo lo que hasta ahora se ha retomado en el transcurso del proceso y la composición de esto en su sentido de vida.

\section{Conclusiones}

La disciplina de la orientación considera fundamental promover la autonomía en cada una de las personas orientadas, en este caso, las madres adolescentes; al desarrollar este aspecto se logra potencializar las fortalezas de la persona, con el fin de que las jóvenes logren afrontar con responsabilidad las situaciones cotidianas dentro del contexto en el que se encuentran y establecer procesos en donde se evidencie cómo estas madres pueden, mediante la automotivación y el reconocimiento que reciben por parte de otras personas, obtener sus logros académicos, sentirse seguras y confiadas de sus capacidades y, con ello, visualizar el aprendizaje como un reto positivo que produce satisfacción.

No obstante, no debe obviarse el hecho de que el embarazo en adolescentes estudiantes implica que las jóvenes realicen cambios significativos en su vida, en cuanto a los gustos e intereses, pues muchos de estos se modifican, o bien, se realizan mientras ellas atienden a sus bebés, además el uso del tiempo y la toma de decisiones se ven fuertemente influenciados por su condición de madre.

Para las jóvenes que formaron parte de la investigación, la principal motivación es llevar a cabo las acciones pertinentes para garantizar el bienestar de su hija o hijo, por ejemplo, una mejor organización del tiempo en función del cuido de su bebé, para el logro académico y su permanencia en la institución educativa. Desde la perspectiva de las jóvenes 
entrevistadas, una mayor preparación académica les permitirá mejores posibilidades de aportar independientemente los recursos necesarios para una buena calidad de vida, de sí mismas y para sus hijas e hijos. En lo referente al reconocimiento de logros académicos, la población presenta dos perspectivas diferentes, un grupo de jóvenes se reconocen a sí mismas y se sienten reconocidas por personas cercanas a ellas y en una situación distinta, otras no cuentan con alguien que les brinde ese tipo de reconocimiento.

Igualmente, la principal razón que da sentido a la vida de la población estudiada son sus niñas y niños, esto, a través de brindar estabilidad en pro del bienestar próximo y futuro de sí mismas y de sus hijas e hijos. Así se favorece la persistencia y continuidad de las jóvenes madres en sus estudios, ya que la formación académica es el medio que ellas conciben necesario para lograr mejores condiciones socioeconómicas. La experiencia de ser madres implicó en las adolescentes realizar ajustes en su rutina diaria, tales como dejar de maquillarse, hacerse más responsable, y estar a cargo del cuidado de sus bebés. Sumado a esto, la maternidad contribuye con la construcción dinámica del sentido de vida de estas, en tanto les proporciona una mayor direccionalidad en su comportamiento y la búsqueda del significado de vida, ese proceso es único y específico en cada una de las madres adolescentes y les genera sentimientos y actitudes que se convierten en una fuerza interna para continuar formándose académicamente.

Las madres adolescentes entrevistadas manifiestan que el asesoramiento brindado está direccionado mayormente a las becas; sin embargo, esas acciones han contribuido a la permanencia de las madres adolescentes en la institución. Finalmente, la orientación debe procurar atender las necesidades de estas adolescentes valorando la condición de cada una. Así, la persona orientadora puede partir de la integración de sus funciones, los procesos de la orientación y los rasgos del sentido vida como factores protectores, con el fin de favorecer la permanencia de las madres adolescentes en los centros educativos.

\section{Referencias}

Andrade-Ruíz, F. y Sánchez-Solano, J. (2000). Adolescentes embarazadas casadas: Un análisis de las tareas básicas de la adolescencia. Revista de Adolescencia y Salud, 2(1), Recuperado de http://www.binasss.sa.cr/revistas/ays/2n1/art3.htm

Arguedas-Negrini, I. (2010). Promoción de la permanencia de estudiantes en la educación secundaria. San José, Costa Rica: INIE.

Badilla-Marín, E., Castro-López, R. Meza-Rodríguez, E., Rodríguez-Aguilar, E., Sibaja-Villalobos, K. y Vargas-Bonilla, E. (2016). Rasgos del sentido de vida que favorecen la permanencia de la madre adolescente en el sistema educativo formal (Tesis de Licenciatura). Universidad de Costa Rica, San José, Costa Rica. 
doi: http://dx.doi.org/10.15359/ree.21-3.8

URL: http://www.una.ac.cr/educare

CORREO: educare@una.cr

Barrantes-Echeverría, R. (2003). Investigación. Un camino al conocimiento. Un enfoque cualitativo y cuantitativo. San José, Costa Rica: EUNED.

Beltrán-Llera, J. y Bueno-Álvarez, J. J. (Eds.). (1995). Psicología educativa. Barcelona: Marcombo.

Caballero-Batista, A. y Méndez-Díaz, T. (2005). El sentido psicológico del embarazo en adolescentes gestantes. Santiago 108, 62-92.

Cartin, A. (2011). Propuesta orientaciones básicas para la atención del embarazo y maternidad en población de personas menores de edad insertas en el sistema educativo. Ministerio de Educación Pública, San José, Costa Rica. Recuperado de http://studylib.es/doc/131650/ propuesta-de-orientaciones-b\%C3\%A1sicas-para-la-atenci\%C3\%B3n-del-e...

Cavallé, M. (2013). El sentido filosófico de la existencia humana. Anthropos. Recuperado de http://www.monicacavalle.com/wordpress/wp-content/uploads/2013/01/El-sentidofilos\%C3\%B3fico-de-la-vida-humana.-Extracto..pdf

Celedón, R y Garri, M. (2014). Familias adolescentes: Entre no ser, no tener y no acceder. Psicoperspectivas Individuo y Sociedad, 13(2), 67-78. doi: https://doi.org/10.5027/ psicoperspectivas-Vol13-Issue2-fulltext-424

Coll,A.(2001).Embarazo en la adolescencia ¿cuáles el problema?EnS.-Burak (Comp.),Adolescencia yjuventuden América Latina (pp.427-429). Cartago, Costa Rica: Libro Universitario Regional. Recuperado de http://www.binasss.sa.cr/adolescencia/Adolescenciayjuventud.pdf

Donas-Burak, S. (2001). Adolescencia y juventud. Viejos y nuevos desafíos en los albores de nuevo milenio. En S. Donas-Burak (Comp.), Adolescencia y juventud en América Latina (pp. 23-29). Cartago, Costa Rica: Libro Universitario Regional. Recuperado de http://www. binasss.sa.cr/adolescencia/Adolescenciayjuventud.pdf

Educarchile. (2014). La importancia de la motivación y el reconocimiento de los logros escolares obtenidos. Recuperado de http://www.educarchile.cl/ech/pro/app/detalle?id=75532

Espada-García, M. (2006). Nuestro motor emocional "La motivación". Con motivación nuestra vida será más estiulante, exitosa y satisfacotria. O no será. Madrid: Ediciones Díaz de Santos. Recuperado de http://site.ebrary.com.ezproxy.sibdi.ucr.ac.cr:2048/lib/sibdilibrosp/reader. action?doc|D=10149751

Frankl, V. (1991). El hombre en búsqueda de sentido (12 ed.). Barcelona: Editorial Herder. Recuperado de https://markeythink.files.wordpress.com/2011/04/el hombre en busca de sentido viktor frankl.pdf 
Fuller-Osores, N. (2001). Maternidad e identidad femenina: Relato de sus desencuentros. En S. (Comp.), Adolescencia y juventud en América Latina (pp. 225-242). Cartago, Costa Rica: Libro Universitario Regional. Recuperado de http://www.binasss.sa.cr/adolescencia/ Adolescenciayjuventud.pdf

Gurdián-Fernández, A. (2007). El paradigma cualitativo en la investigación socio-educativa (Colección IDER). San José, Costa Rica: CECC Y AECI. Recuperado de https://web.ua.es/ en/ice/documentos/recursos/materiales/el-paradigma-cualitativo-en-la-investigacionsocio-educativa.pdf

Hernández-Sampieri, R. Fernández-Collado, C. y Baptista-Lucio, P. (2003). Metodología de la Investigación. Colombia: McGraw-Hill.

Instituto Nacional de Estadísticas y Censos (INEC). (2013). Estadísticas vitales 2012. San José, Costa Rica. Recuperado de http://www.inec.go.cr/sites/default/files/documentos/poblacion/ fecundidad/estadisticas/resultados/repoblacev2012-04.xls

Issler, J. R. (2001). Embarazo en la adolescencia. Revista de Posgrado de la Cátedra Via Medicina, 107, 11-23. Recuperado de http://med.unne.edu.ar/revista/revista107/emb adolescencia. $\underline{\mathrm{html}}$

Jiménez-Segura, F. y Arguedas-Negrini, I. (2004). Rasgos de sentido de vida del enfoque de resiliencia en personas mayores en los 65 y 75 años. Revista Actualidades Investigadas en Educación, 4(2), 1-28. Recuperado de http://revistas.ucr.ac.cr/index.php/aie/article/ view/9090/17488

Krauskopf-Roger, D. (1994). Adolescencia y educación (2a ed.). San José, Costa Rica: EUNED.

Krauskopf-Roger, D. (2011). El desarrollo en la adolescencia: Las transformaciones psicosociales y los derechos en una época de cambios. Revista Psicologia.com, 15, 15-51. Recuperado de http://hdl.handle.net/10401/4562

Loaiza-Valdés, O. (2005). Construcción del sentido de vida en jóvenes universitarias (Tesis doctoral). Universidad Hiberoamericana. Distrito Federal, México. Recuerado de http://www.bib.uia. mx/tesis/pdf/014533/014533.pdf

Lukas, E. (2003). Logoterapia. La búsqueda de sentido. Barcelona: Paidós.

Ministerio de Educación Pública (MEP). (Marzo, 2015). Estudiantes embarazadas y estudiantes madres en centros educativos costarricenses, curso lectivo 2013. Boletín 03, 1-20. Recuperado de http://www.mep.go.cr/indicadores edu/BOLETINES/03 15.pdf

Nava-Flores, C. M. (2009). La maternidad en la adolescencia. Contribuciones a las ciencias sociales. Recuperado de www.eumed.net/rev/cccss/06/cmnf.htm 
Noblejas-De la Flor, M. Á. (2000). Palabras para una vida con sentido (2a ed.). Bilbao: Desclée de Breuwer.Recuperado de http://www.cpalsj.org/wp-content/uploads/2014/08/14CPM3T3Noblejas-2000-Palabras-para-una-vida-con-sentido.pdf

Ortiz-Arias, G. (2012). Sentido de vida y narrativa: Defensa frente a la apatía del hombre para pensar lo humano. Katharsis, 13, 107-124. doi: http://dx.doi.org/10.25057/25005731.437

Paz-López, M. (2005). La práctica del orientador educativo y la motivación de logro en los alumnos. Revista Mexicana de Orientación Educativa, 3(6), 31-37. Recuperado de http:// www.remo.ws/REVISTAS/remo-6.pdf

Quintero-Rondón, A. P. y Rojas-Betancur, H. M. (2015). El embarazo a temprana edad, un análisis desde la perspectiva de madres adolescentes. Revista Virtual Universidad Católica del Norte, 44, 222-237. Recuperado de http://revistavirtual.ucn.edu.co/index.php/RevistaUCN/ article/view/626/1161

Rojas-Valenciano, L. P. (2008). Elementos conceptuales y metodológicos de la investigación cualitativa. San José, Costa Rica: Editorial UCR.

Santrock, J. (2002). Psicología de la educación. México: McGraw-Hill.

Secretaría de Seguridad Pública, Gobierno Federal de los Estados Unidos Mexicanos. (2011). Deserción escolar y conductas de riesgo en adolescentes. México: Autor. Recuperado de http://telesecundaria.gob.mx/mesa tecnica/files/Desercion-Escolar.pdf

Taylor, S. J. y Bogdan, R. (1987). Introducción a los métodos cualitativos de investigación (2a ed.). Barcelona: Paidós.

Wong, P. P. y Wong, L. C. J. (2011). A meaning-centered approach to building youth resilience. Recuperado de http://www.drpaulwong.com/documents/HQM2-chapter27.pdf 